\title{
Notas sobre la ecología reproductiva de árboles en un bosque mesófilo de montaña en Michoacán, México
}

\author{
HéCTOR M. HeRNÁNDEZ ${ }^{1,2}$ y Yasmín CARReÓN ABUd ${ }^{1}$
}

A la memoria de Pepe Cué y José Miguel Torre

H.M.H.

\begin{abstract}
RESUMEN. Se describen algunos eventos reproductivos de los principales elementos arbóreos de un bosque mesófilo de montaña, y se complementa esta información con datos sobre algunas especies arbustivas y herbáceas. La floración de los árboles resultó ser fuertemente estacional, con un mercado pico en las interfases sequía-lluvias y lluvias-sequía, mientras que las especies arbustivas y herbáceas florecieron básicamente durante la estación húmeda. Todas las especies arbóreas, con excepción de Cornus disciflora, poseen niveles relativamente altos de autocompatibilidad. Se considera que la elevada incidencia de este sistema de reproducción es un mecanismo adaptativo que aumenta las posibilidades de éxito reproductivo de las especies en ausencia de polinizadores eficientes. La melitofilia es el síndrome de polinización más común, aunque existen otros representados como anemofilia, ornitofilia y esfingofilia. Finalmente se discuten los rasgos reproductivos generales del bosque, y se comparan con la naturaleza reproductiva de comunidades vegetales de climas templados y tropicales.
\end{abstract}

ABSTRACT. Reproductive events of the main tree species of a mountain mesophytic forest are described, and complemented with data on the reproductive behavior of some shrubs and herbs. The general pattern of flowering among tree species was strongly seasonal, with a clear peak in flowering during the dry-wet and wet-dry interfases. Shrubs and herbs, by contrast, flowered primarily during the rainy season. Controlled pollination experiments showed that all tree species, with the exception of Cornus disciflora, have relatively high levels of self-compatibility. This is considered a mechanism for ensuring the reproductive success of these species in the absence of adequate pollinators. Melittophily is the dominant pollination syndrome, although others were observed (e.g., anemophily, ornithophily, and sphingophily). Finally, the general reproductive features of the forest are discussed and compared with the reproductive behavior of temperate and tropical forests.

${ }^{1}$ Escuela de Biología, Universidad Michoacana de San Nicolás de Hidalgo, 58020 Morelia, Mich., Méxịco.

${ }^{2}$ Dirección actual: Departamento de Botánica, Instituto de Biología, Universidad Nacional Autónoma de México, Apdo. Postal 70-233, 04510 México, D.F. 
Recientemente se ha dado mucha atención a la diversidad de fenómenos ligados con la biología reproductiva de las plantas (Janzen, 1967; Bawa, 1974; Gentry, 1974; Frankie, et al, 1974; Ruiz y Kalin, 1978 y muchos otros). Sin embargo, en nuestro país el conocimiento sobre estos aspectos es todavía incipiente, a pesar de la importancia que revisten para el entendimiento de la ecología y la evolución de las especies vegetales. Este estudio tiene como objetivo global contribuir al entendimiento de los patrones de organización de los principales elementos arbóreos de un bosque mesófilo de montaña, poniendo especial énfasis en los siguientes eventos: 1) fenología floral, 2) sistemas de reproducción, y 3) relaciones planta-polinizador.

\section{ÁREA DE ESTUDIO Y MÉTODOS}

El trabajo de campo se desarrolló en su totalidad en un sitio permanente de observación ubicado a $7.8 \mathrm{~km}$ al sur de Pátzcuaro, Michoacán, el cual se encuentra enclavado en la Cordillera del Eje Neovolcánico a $2350 \mathrm{msnm}$. De acuerdo con los datos de la Estación Meteorológica de Pátzcuaro, el área se encuentra afectada por un clima templado, con una temperatura media anual de $16.4^{\circ} \mathrm{C}$; las temperaturas máxima y mínima registradas son de $37^{\circ}$ y $-5^{\circ} \mathrm{C}$, respectivamente. La precipitación media anual es de 1041.2 $\mathrm{mm}$., siendo en el periodo de fines de mayo a octubre cuando se registra la mayor cantidad de lluvias. La estación de sequía, por otra parte, coincide con el período en el que se registran las temperaturas más bajas (fig. 1).

En el área de estudio se desarrolla, sobre todo en los sitios protegidos por el viento y la fuerte insolación, un bosque mesófilo de montaña relativamente bien conservado. Este sitio abarca un terreno de 500 hectáreas, el cual recientemente fue declarado área de protección de la flora y la fauna. El bosque mesófilo de montaña, famoso por sus afinidades fitogeográficas con el del este de los Estados Unidos, ha sido caracterizado por Rzedowski $(1970,1978)$ y por Rzedowski y McVaugh (1966) como un tipo de vegetación de distribución discontinua, que se desarrolla en sitios montañosos de clima templado y en donde las condiciones de humedad son favorables.

Se estudiaron en detalle las especies Arbutus xalapensis H.B.K., Ternstroemia pringlei Standl., Tilia mexicana Schlecht., Styrax ramirezii Greenm., Clethra mexicana DC., Cornus disciflora DC. y Symplocos prionophylla Hemsl., las cuales, además de Quercus rugosa Nee, Q. laurina Humb. et Bonpl. y Pinus pseudostrobus Lindl., son los elementos más abundantes del sitio de observación. Se presentan también algunos resultados adicionales sobre especies pertenecientes a otros estratos del bosque.

Las observaciones se realizaron en un área de aproximadamente 20-30 hectáreas, de enero de 1980 a diciembre de 1981, en donde se hicieron visitas ininterrumpidas a intervalos de 15 días en promedio. Para conocer la dinámica de la floración de los árboles, se marcaron 10 individuos de cada especie, sobre los cuales se hicieron observaciones periódicas mensuales. De esta forma se llevó un diagnóstico detallado del comportamiento fenológico de cada una de las especies. Dada la dificultad para evaluar cuantitativamente la intensidad de la floración de los individuos, se utilizaron tres categorías diferentes, con las cuales se obtuvo una idea aproximada, de la dinámica de la floración de la comunidad de árboles. Dichas categorías son las siguientes: o = floración nula; 1 = floración cercana al $50 \%$ de la intensidad esperada, y $2=$ floración cercana al $100 \%$. Sobre estas 


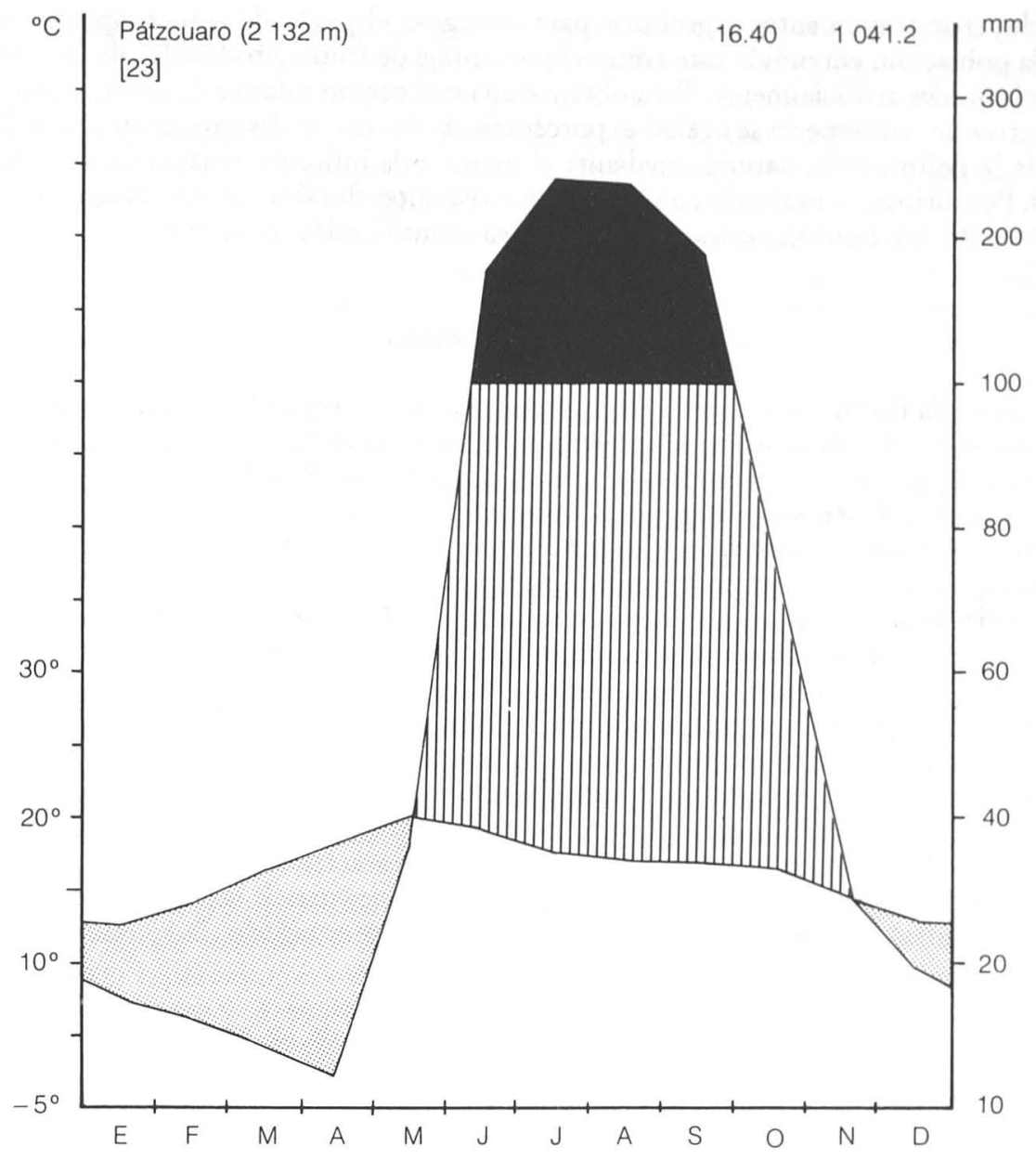

Fig. 1. Diagrama climático de la Estación Meteorológica de Pátzcuaro. El área sombreada indica la estación seca; la rayada la estación húmeda y la negra los meses en los que se registran más de $100 \mathrm{~mm}$ de precipitación. Temperatura media anual $=16.4^{\circ} \mathrm{C}$; precipitación media anual $=1041.2 \mathrm{~mm}$; temperatura mínima absoluta $=-5^{\circ} \mathrm{C}$. Para información adicional sobre la construcción e interpretación de este diagrama consúltese Walter (1973, pp. 25-30).

bases, generalmente consideramos una especie en floración cuando al menos la mitad de los individuos marcados se encontraban en la categoría 1 o 2. Este criterio, sin embargo, no se utilizó en varios casos debido a que la floración de algunas especies resultó sumamente asincrónica.

En combinación con lo anterior, y para conocer los sistemas de reproducción de las especies, se emplearon las técnicas experimentales descritas por Bawa (1974), las cua- 
les incluyeron tratamientos específicos para averiguar el grado de autocompatibilidad de cada población, entendido éste como el porcentaje de frutos producidos de las flores autopolinizadas artificialmente. Para obtener una estimación relativa de la eficiencia reproductiva de cada especie se evaluó el porcentaje de flores que desarrollaron frutos después de la polinización natural, mediante el marcaje de inflorescencias o flores individuales. Por último, se evaluó la conducta de los visitantes florales por observación directa $y$, cuando fue posible, se les colectó para su identificación posterior.

\section{RESULTADOS Y DISCUSIÓN}

Fenología floral. De la tabla 1 se desprende que en las especies arbóreas del bosque estudiado se pueden distinguir cuatro patrones temporales de floración. En primer lugar las especies que florecen en medio, o hacia la segunda mitad de la estación de sequía, como es el caso de Arbutus xalapensis, Clethra mexicana, Cornus disciflora, Eupatorium mairetianum, Pinus pseudostrobus, Quercus rugosa y Q. laurina. El segundo patrón incluye a las especies que comienzan su período de floración en la estación seca y la extienden hasta iniciadas las lluvias, como Ternstroemia pringlei, Tilia mexicana, y Stryrax ramirezii. En tercer lugar está Oreopanax xalapensis que florece exclusivamente durante la estación húmeda, y finalmente Meliosma dentata y Symplocos prionophylla que empiezan a florecer en esta estación y siguen produciendo flores hasta el inicio del período de sequía.

De particular interés es que 12 de las 13 especies registradas en la tabla 1 ubican sus picos de floración en la estación seca. En este sentido, es importante mencionar la hipótesis de Janzen (1967), quien discute la posibilidad de que la sincronización de los períodos de floración en esta época del año tiene un carácter adaptativo debido a que la estación de sequía está asociada con una serie de ventajas para la actividad reproductiva de las plantas. En efecto, como se observó en el sitio estudiado, la actividad de los

TABLA 1. Comportamiento estacional de la floración de las especies arbóreas

(Datos acumulados de los años 1980-1981)

\begin{tabular}{|c|c|c|c|c|c|c|c|c|c|c|c|c|}
\hline Especie entomófila & $\mathrm{E}$ & $\mathrm{F}$ & M & A & $\mathrm{M}$ & $\mathrm{J}$ & $\mathrm{J}$ & A & $\mathrm{S}$ & $\mathrm{O}$ & $\mathrm{N}$ & $\mathrm{D}$ \\
\hline $\begin{array}{l}\text { Arbutus xalapensis } \\
\text { Clethra mexicana } \\
\text { Cornus disciflora } \\
\text { Eupatorium mairetianum* } \\
\text { Ternstroemia pringlei } \\
\text { Tilia mexicana } \\
\text { Styrax ramirezii } \\
\text { Oreopanax xalapensis } \\
\text { Meliosma dentata } \\
\text { Symplocos prionophylla } \\
\end{array}$ & - & 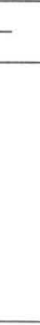 & 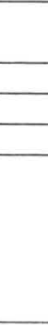 & - & & - & & 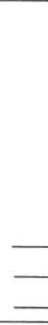 & & & & \\
\hline Especies anemófilas & $E$ & $F$ & $M$ & $A$ & $M$ & $J$ & $J$ & $A$ & $S$ & $\mathrm{O}$ & $N$ & $D$ \\
\hline $\begin{array}{l}\text { Quercus laurina } \\
\text { Q. rugosa } \\
\text { Pinus pseudostrobus }\end{array}$ & & & & & & & & & & & & \\
\hline
\end{tabular}

*Arbusto. 
polinizadores potenciales se ve fuertemente reducida por las lluvias, además de que éstas pueden tener efectos destructivos sobre las flores. Es posible que los insectos antófilos, que presentan una mayor actividad antes de las lluvias, hayan actuado como fuerzas selectivas en el desarrollo de este patrón fenológico de la comunidad.

En lo que respecta a las especies anemófilas, la ventaja de que florezcan durante la estación seca resulta evidente, debido a que la dispersión de la enorme cantidad de granos de polen (Faegri y van der Pijl, 1979) se vería minimizada por las lluvias (Whitehead, 1969). Pinus pseudostrobus representa una excepción al patrón general seguido por las especies anemófilas ya que, aunque su pico de floración se ubica en la estación seca, pequeñas cantidades de microestróbilos se producen continuamente hasta el mes de septiembre.

Otro aspecto asociado a la fenología de los árboles es la secuencia de sus períodos de floración (tabla 1). Este comportamiento se ha observado en comunidades templadas (Heinrich, 1976; Mosquin, 1971) y tropicales (Gentry, 1974; Frankie et al. 1974), y existe el consenso de que el escalonamiento de los períodos de floración de las especies que cohabitan en una comunidad dada ha sido probablemente el resultado de las presiones selectivas que produce la competencia por los servicios de polinizadores específicos (Mosquin, 1971; Levin y Anderson, 1970). En este sentido, es importante mencionar que también existe un notable desfasamiento entre las épocas de floración de los árboles y las especies herbáceas y arbustivas entomófilas que se encuentran en el sitio de estudio. Una gran cantidad de hierbas y arbustos florecen en la estación húmeda, lo cual marca una clara diferenciación entre los nichos temporales que éstas ocupan de acuerdo con sus épocas de floración, la que, a su vez, se traduce en una mayor disponibilidad de recursos energéticos, en forma de néctar y polen, para los polinizadores que dependen de la comunidad.

Finalmente, es importante mencionar que es posible distinguir dos estrategias diferentes en lo que se refiere a la conducta de floración de los árboles, las cuales tienen importantes repercusiones sobre la conducta de los visitantes. Tilia mexicana, Eupatorium mairetianum y Arbutus xalapensis ilustran una de estas estrategias, ya que individuos de estas especies producen una gran cantidad de flores relativamente generalizadas, las cuales son visitadas por una variedad de insectos durante períodos prolongados. Contrastando con esta estrategia de floración explosiva, existe una segunda modalidad, ilustrada por Ternstroemia pringlei y Symplocos prionophylla, que consiste en la producción de pequeñas cantidades de flores por individuo por un período relativamente extendido. Este racionamiento de flores en antesis implica que los visitantes de estas especies se vean obligados a desplazarse de un individuo a otro para satisfacer sus requerimientos energéticos (Heinrich y Raven, 1972). Esta conducta de desplazamiento, designada como conducta nómada de forrajeo o "trap-line" (Janzen, 1971), maximiza la polinización cruzada. Estas modalidades de floración han sido discutidas previamente por Frankie et al. (1974), Gentry (1974), Janzen (1971) y otros.

Sistemas de reproducción. Los resultados de los sistemas de reproducción de los árboles muestran una alta proporción de especies autocompatibles, dado que cinco de las seis especies de las que se obtuvieron datos cuantitativos poseen un alto grado de autocompatibilidad (tabla 2). Además, hay razones para afirmar que Oreopanax xalapen. sis y Meliosma dentata son autógamas, ya que individuos aislados produjeron frutos aun- 
TABLA 2. Resultados de los experimentos de polinización controlada de las especies arbóreas

\begin{tabular}{|c|c|c|c|c|c|c|}
\hline \multirow[b]{2}{*}{ Especie } & \multirow{2}{*}{$\begin{array}{c}\text { Núm. de individuos } \\
\text { tratados }\end{array}$} & \multicolumn{2}{|c|}{ Núm. de flores } & \multicolumn{2}{|c|}{$\%$ de flores de frutos } & \multirow[b]{2}{*}{ Conclusión } \\
\hline & & Autopolinizadas & Cruzadas & Autopolinizadas & Cruzadas & \\
\hline Arbutus xalapensis & 6 & 133 & 39 & 23 & 74.3 & $\mathrm{AC}$ \\
\hline Ternstroemia pringlei & 6 & 10 & 12 & 100 & 66.6 & $\mathrm{AC}$ \\
\hline Tilia mexicana & 3 & 45 & 14 & 93.3 & 100 & $\mathrm{AC}$ \\
\hline Clethra mexicana & 3 & 32 & 13 & 78.1 & 69.6 & $\mathrm{AC}$ \\
\hline Symplocos prionophylla & 5 & 27 & 26 & 11.1 & 42.3 & $\mathrm{AC}$ \\
\hline Cornus disciflora & 10 & 55 & 41 & 0 & 36.5 & $\mathrm{AI}$ \\
\hline
\end{tabular}

"AC $=$ autocompatible; $\mathrm{AI}=$ autoincompatible.

que sus inflorescencias fueron aisladas de la actividad de los polinizadores mediante bolsas de polinización; sin embargo, para demostrar la existencia de este sistema de reproducción en estas especies se requiere de trabajo adicional. Por otra parte, la evaluación de los sistemas de reproducción de algunas especies herbáceas y arbustivas del sitio muestra el mismo patrón, ya que la mayor parte de ellas resultó ser autocompatible (tabla 3).

Este comportamiento contrasta con el registrado para comunidades tropicales de Centro y Sudamérica. En efecto, Bawa (1974) y Ruiz y Kalin (1978) encontraron que la mayoría de las especies que habitan en estas comunidades son exógamas obligadas gracias a la posesión mecanismos de autoincompatibilidad y dioecia. Sin embargo, Primak y Tomlison (1980), en una nota sobre los sistemas de reproducción de especies componentes de manglares, proponen que la autocompatibilidad puede ser común en estas comunidades; Pojar (1974) informa que existe un predominio de especies autocompatibles en tres diferentes tipos de vegetación del Canadá. Más aun, Kalin y Cabrera (1978); en una evaluación preliminar sobre sistemas de incompatibilidad de especies de un bosque nublado en Venezuela, un tipo de vegetación estructuralmente similar al bosque mesófilo de montaña, encuentran una frecuencia de $50 \%$ de especies autocompatibles.

La autocompatibilidad frecuentemente se asocia a especies características de la sucesión secundaria, sobre todo a las pioneras que crecen en las etapas tempranas de la suce-

TABLA 3. Resultados de los experimentos de polinización controlada de algunas especies arbustivas y herbáceas

\begin{tabular}{|c|c|c|c|c|c|c|}
\hline \multirow[b]{2}{*}{ Especie } & \multirow{2}{*}{$\begin{array}{l}\text { Núm. de individuos } \\
\text { tratados }\end{array}$} & \multicolumn{2}{|c|}{ Núm. de flores } & \multicolumn{2}{|c|}{$\%$ de frutos de flores } & \multirow[b]{2}{*}{ Conclusión } \\
\hline & & Autopolinizadas & Cruzadas & s Autopolinizadas & Cruzadas & \\
\hline Salvia elegans & 11 & 17 & 7 & 58.8 & 85.7 & $\mathrm{AC}$ \\
\hline S. purpurea & 5 & 31 & 19 & 48.38 & 57.9 & $\mathrm{AC}$ \\
\hline S. mexicana & 15 & 29 & 16 & 44.8 & 68.7 & $\mathrm{AC}$ \\
\hline Agastache mexicana & 5 & 33 & 46 & 78.7 & 71.3 & $\mathrm{AC}$ \\
\hline Castilleja tenuiflora & 3 & 9 & 5 & 88.9 & 80 & $\mathrm{AC}$ \\
\hline C. arvensis & 22 & 55 & 46 & 1.8 & 95.6 & AI \\
\hline Fuchsia fulgens & 7 & 15 & 4 & 46.6 & 50 & $\mathrm{AC}$ \\
\hline
\end{tabular}

"AC= autocompatible; $\mathrm{AI}=$ autoincompatible. 
sión (Opler et al. 1980). Una razón que nos ayuda a explicar este hecho es que la impredecibilidad de polinizadores eficientes en este tipo de ambientes ha funcionado como fuerza selectiva para el desarrollo de los sistemas de autocompatibilidad. En este sentido, Levin (1973) propone que otro factor que puede estimular la evolución de la autogamia es la competencia por los servicios de los polinizadores. En relación con lo anterior es evidente que el área de estudio presenta signos de disturbio, como lo demuestra la abundancia de individuos de Eupatorium mairetianum y Ternstroemia pringlei, además de la existencia de estratos herbáceo y arbustivo bien definidos en algunos sitios, un rasgo inesperado cuando el bosque mesófilo de montaña no ha sido alterado (Rzedowski, 1970, 1978; Rzedowski y McVaugh, 1966).

Una característica reproductiva de los árboles que confirma el fuerte predominio de la autocompatibilidad, son los altos porcentajes de frutos producidos por las especies autocompatibles como resultado de la polinización natural. En efecto, como se muestra en la tabla 4, las especies con este sistema de reproducción producen cantidades relativamente altas de frutos, en comparación con Cornus disciflora que es la única especie autoincompatible. Estos resultados concuerdan con los encontrados por Bawa (1974) en Costa Rica, quien opina que la ventaja de la autocompatibilidad consiste en el incremento de las posibilidades de fertilización. Styrax ramirezii plantea un problema a resolver, ya que en los dos años consecutivos en los que se marcaron sus flores no se registraron frutos (tabla 4). Una observación interesante en este sentido es que los individuos utilizados para esta evaluación, relativamente jóvenes, produjeron flores, mas no frutos; sin embargo, pudimos notar que sólo algunos árboles, aparentemente viejos, desarrollaron cantidades regulares de frutos.

Visitantes florales. De un análisis de las características florales de las especies arbóreas que integran la comunidad se desprende que la entomofilia es el síndrome de polinización dominante. En lo que respecta a los estratos arbustivo y herbáceo, existen especies entomófilas, ornitófilas y anemófilas, con un claro predominio de las primeras.

Aunque, como discutimos anteriormente, la mayor parte de las especies poseen altos grados de autocompatibilidad, es posible afirmar en términos generales que son básicamente exógamas. Como se muestra en la tabla 5, las flores de las diferentes especies son una importante fuente de energía para especies correspondientes a cinco órdenes

TABLA 4. Porcentajes de flores que produjeron frutos

a partir de polinización natural

\begin{tabular}{|c|c|c|c|c|c|}
\hline \multirow[b]{2}{*}{ Especie } & \multirow{2}{*}{$\begin{array}{l}\text { Sistema de } \\
\text { reproducción" }\end{array}$} & \multicolumn{2}{|c|}{1980} & \multicolumn{2}{|c|}{1981} \\
\hline & & $\%$ & $\overline{N^{*}}$ & $\%$ & $\mathrm{~N}$ \\
\hline Arbutus xalapensis & $\mathrm{AC}$ & 91.3 & 395 & - & - \\
\hline Ternstroemia pringlei & AC & 63 & 97 & 50.5 & 97 \\
\hline Tilia mexicana & AC & 54 & 37 & - & - \\
\hline Clethra mexicana & $\mathrm{AC}$ & - & - & 38.8 & 369 \\
\hline Oreopanax xalapensis & $\mathrm{AC}$ & - & - & 97.9 & 288 \\
\hline Styrax ramirezii & $\mathrm{AC}$ & 0 & 90 & 0 & 95 \\
\hline Cornus disciflora & $\mathrm{AI}$ & 6.9 & 582 & 6.1 & 899 \\
\hline
\end{tabular}

* $\mathrm{AC}=$ autocompatible; $\mathrm{AI}=$ autoincompatible; $\mathrm{N}=$ número de flores marcadas. 
TABLA 5. Visitantes florales de las especies arbóreas

\begin{tabular}{|c|c|c|c|c|c|c|c|c|}
\hline Visitante & $\begin{array}{l}\text { Arbutus } \\
\text { xalapensis }\end{array}$ & $\begin{array}{l}\text { Ternstroemia } \\
s \quad \text { pringlei }\end{array}$ & $\begin{array}{c}\text { Cornus } \\
\text { disciflora }\end{array}$ & $\begin{array}{c}\text { Tilia } \\
\text { mexicana }\end{array}$ & $\begin{array}{l}\text { Styrax } \\
\text { ramirezii }\end{array}$ & $\begin{array}{c}\text { Meliosma } \\
\text { dentata }\end{array}$ & $\begin{array}{l}\text { Eupatorium* } \\
\text { mairetianum }\end{array}$ & $\begin{array}{c}\text { Symplocos } \\
\text { prio. } \\
\text { nophylla }\end{array}$ \\
\hline $\begin{array}{l}\text { Aves: Trochilidae } \\
\text { Lampornis cle- } \\
\text { menciae }\end{array}$ & Xc & & & & & & & \\
\hline $\begin{array}{l}\text { Hymenoptera } \\
\text { Bombus ephipiatus form } \\
\text { Xylocopa sp. } \\
\text { Apis mellifera }\end{array}$ & Xosus & $\mathrm{Xc}_{\mathrm{c}}$ & $\mathrm{X}_{\mathrm{c}}$ & Xc & Xc & & $\begin{array}{l}\mathrm{Xc} \\
\mathrm{Xc}\end{array}$ & Xc \\
\hline $\begin{array}{l}\text { Lepidoptera } \\
\text { Danaus plexippus } \\
\text { Catasticta nimbice } \\
\text { Urbanus sp. } \\
\text { Apicia sp. } \\
\text { Noctuidae } \\
\text { sp. no identificada }\end{array}$ & & $\begin{array}{l}\text { Xo } \\
\text { Xo }\end{array}$ & & $\begin{array}{l}\text { Xo } \\
\text { Xo } \\
\text { Xc } \\
\text { Xc }\end{array}$ & $\begin{array}{l}\mathrm{Xc} \\
\mathrm{Xc}\end{array}$ & & $\mathrm{Xc}$ & \\
\hline $\begin{array}{l}\text { Diptera } \\
\text { Tachinidae } \\
\text { spp. no identificadas }\end{array}$ & & & & $\mathrm{Xc}_{\mathrm{c}}$ & Xc & Xo & & \\
\hline $\begin{array}{l}\text { Coleoptera } \\
\text { Atheta discrepans } \\
\text { Colopterux sp. } \\
\text { Phyllotrox sp. } \\
\text { sp. no identificada }\end{array}$ & & $\begin{array}{l}\text { Xo } \\
\text { Xo } \\
\text { Xo }\end{array}$ & & & & & & $\mathrm{Xc}_{\mathrm{c}}$ \\
\hline $\begin{array}{l}\text { Thysanoptera } \\
\text { Frankliniella rostrata } \\
\text { F. fallaciosa } \\
\text { F. tridacana } \\
\text { Taeniotbrips tibialis } \\
\text { T. mexicanus }\end{array}$ & $\begin{array}{l}\mathrm{Xc} \\
\mathrm{Xc} \\
\mathrm{Xc} \\
\mathrm{Xc} \\
\mathrm{Xc}\end{array}$ & $\begin{array}{l}\mathrm{Xc} \\
\mathrm{Xc}_{\mathrm{c}} \\
\mathrm{X}_{\mathrm{c}} \\
\mathrm{Xc}\end{array}$ & & & & & & \\
\hline
\end{tabular}

$\mathrm{X} c=$ visitante común; $\mathrm{Xo}_{\mathrm{o}}=$ visitante ocasional; $*$ = arbusto.

de insectos. El más activo de éstos resultó ser Bombus ephipiatus formosus (Hymenoptera) y, en menor medida, varias especies de lepidópteros diurnos y algunos dípteros. Por otra parte, nos fue posible observar individuos de varias especies de pequeños coleópteros que habitan en el interior de las flores de Ternstroemia pringlei y Symplocos prionophylla; sin embargo, es difícil evaluar el papel que desempeñan en la reproducción de las plantas.

De particular interés es el hallazgo de tisanópteros de los géneros Frankliniella y Taeniothrips en las flores de Arbutus xalapensis y Ternstroemia pringlei. Estos pequeños insectos son probablemente elementos importantes en la polinización, debido a su prolongada actividad en el interior de las flores, las cuales utilizan como refugio y alimento. Vale la pena mencionar que especies correspondientes a estos dos géneros de tisanópteros han sido considerados por Thien (1980), Hagerup y Hagerup (1953) y Hagerup (1950) como importantes polinizadores de una variedad de especies en el Viejo Mundo. Así, 
parece probable que estos artrópodos, generalmente no considerados, son importantes agentes de polinización.

El hecho de que algunas especies sean visitadas por tal variedad de animales sugiere que sus flores son morfológica y conductualmente generalizadas en relación con sus mecanismos de polinización, como es el caso de Styrax ramirezii y Eupatorium mairetianum. Sin embargo, Tilia mexicana ilustra un caso extremo ya que, además de las especies de himenópteros, dípteros y lepidópteros diurnos que visitaron recurrentemente sus flores, se observaron varias especies de lepidópteros nocturnos, algunos de los cuales estuvieron en actividad durante el día y hasta las 23:00 horas aproximadamente. En contraste, no fue registrado ningún visitante en las flores de Clethra mexicana, una especie que por sus rasgos florales parece ser típicamente entomófila. Existen evidencias de que los individuos de la población estudiada son autógamos, debido a que $30.6 \%$ de un total de 377 flores protegidas de la actividad de los visitantes formaron frutos normales. Notablemente este valor sólo varió en dos décimas en relación con el registrado en flores que no fueron aisladas de los polinizadores (ver tabla 4). Esta afirmación concuerda con el sistema de reproducción de la especie (autocompatible) y con la topografía de los órganos florales, ya que la superficie estigmática está comúnmente en contacto con las anteras cuando las flores se encuentran en antesis.

\section{CONSIDERACIONES FINALES}

Un hecho bien establecido es que los bosques de clima templado de México incluyen una combinación de elementos florísticos de afinidades neárticas y neotropicales. En este sentido, el bosque mesófilo de Pátzcuaro, y seguramente la mayoría de los bosques de clima templado del país, están ubicados en una posición intermedia en cuanto a su comportamiento reproductivo. En efecto, mientras las comunidades vegetales de clima templado poseen elementos básicamente enemófilos y entomófilos (Pojar, 1974; Heinrich, 1976), los bosques típicamente tropicales incluyen una enorme diversidad de síndromes de polinización biótica, caracterizados por lo intrincado de sus interrelaciones (Frankie et al. 1974; Gentry, 1974; Ruiz y Kalin, 1978).

En el bosque estudiado no se presentan los variados mecanismos de polinización de los bosques tropicales. Aunque existe un predominio de especies entomófilas como en el trópico, hay una muy importante carencia de sistemas de polinización que involucran vectores que requieren un alto consumo de energía durante el forrajeo, como esfíngidos, colibríes y murciélagos. Si bien es cierto que existe un contingente más o menos numeroso de especies ornitófilas, nuestras observaciones mostraron que la mayoría de éstas depende en ciertas circunstancias de la autogamia en su éxito reproductivo. Un ejemplo representativo es el de Fuchsia fulgens, que es fuertemente autógama, y de la cual nunca se registraron visitantes florales. Por otra parte, la esfingofilia, un síndrome de polinización muy conspicuo en bosques tropicales (Haber y Frankie, 1984) está pobremente representado de Pátzcuaro. Fuimos capaces de detectar solamente dos especies claramente esfingófilas: Mirabilis longiflora L. y Hymenocallis harrisiana Herb. [=Pancratium harrissianum (Herb.) Mat.]. Sin embargo, observaciones detalladas sobre M. longiflora (Hernández, observación personal) mostraron que los individuos de esta población producen una alta proporción de sus frutos a través de la autofertilización. 
Otro aspecto reproductivo que también resultó estar en una posición intermedia entre los bosques de clima templado y tropical es la amplitud de floración de la comunidad o, dicho de otra forma, el intervalo en el que existe al menos una especie en floración en la comunidad completa. Como es de esperar, dada la severidad climática, los bosques de clima templado tienen un período relativamente limitado de crecimiento reproductivo, como se desprende de los datos producidos por Pojar (1974) y Heinrich (1976) para comunidades de Canadá y Estados Unidos respectivamente. En contraste, como han mostrado numerosos estudios fenológicos (Frankie et al. 1974; Gentry, 1974; Janzen, 1967, y muchos otros), las comunidades vegetales tropicales, por su gran diversidad de nichos y su relativa estabilidad climática, presentan una sucesión ininterrumpida de especies en floración a lo largo del año. En este sentido, el bosque mesófilo de Pátzcuaro se aproxima a lo que ocurre en el trópico, con la salvedad de que no existe la diversidad de estrategias de floración (Gentry, 1974), y de que el número de especies floreciendo en un momento dado es sustancialmente menor.

Finalmente, es importante destacar que las especies que integran el bosque estudiado están adaptadas preferencialmente a la xenogamia. El potencial de autocompatibilidad genética funciona como un soporte adaptativo en circunstancias en que la disponibilidad de polinizadores es minimizada.

AgRadecimientos. Los autores expresamos nuestro agradecimiento a Alina Chacón H. por su colaboración a lo largo del estudio; a los estudiantes Irene Ávila, Guadalupe Nieto y Héctor M. Anguiano por su valiosa ayuda en el trabajo de campo; al Dr. Carlos Beutelspacher, Dr. Roberto Johansen, M. en C. Santiago Zaragoza y Biól. Juan M. Labougle por su colaboración en la identificación de los insectos polinizadores y a Luís G. Hernández por la preparación de la figura 1. En forma especial damos las gracias al Dr. A. Gentry por sus atinadas observaciones al manuscrito final y al Ing. Jesús Sepúlveda por su generosidad al permitirnos llevar a cabo la investigación en el bosque de su propiedad. Trabajo subsidiado en su fase final por el Programa Nacional Indicativo de Ecología del Consejo Nacional de Ciencia y Tecnología.

\section{LITERATURA CITADA}

BAWA, K.S. 1974. Breeding systems of tree species of a lowland tropical community. Evolution 28:85-92.

FAegri, K. y L. VAN DER PIJL. 1979. The principles of pollination ecology. 3a. ed., Pergamon Press, Londres.

FRANKIE, G.W., H.G. BAKER y P.A. OpLER. 1974. Comparative phenological studies of trees in tropical wet and dry forests in the lowlands of Costa Rica. J. Ecol. 62:881-919.

Gentry, A.H. 1974. Flowering phenology and diversity in tropical Bignoniaceae. Biotropica 6:64-68.

HABER, W.A. y G.W. FRANKIE. 1984. Characteristics and organization of a tropical hawkmoth community. Biotropica (en prensa).

Hagerup, E. y O. Hagerup. 1953. Thrips pollination of Erica tetralis. New Phytol. 52:1-7.

Hagerup, O. 1950. Thrips pollination of Colluna. Dan. Biol. Medd. 18:1-16.

HEINRICH, B. Y P.H. RAVEN. 1972. Energetics and pollination ecology. Science 176:597-602. 1976. Flowering phenologies: bog, woodland, and disturbed habitats. Ecology 57:890-899.

JANZEN, D.R. 1967. Synchronization of sexual reproduction of trees within the dry season in Central America. Evolution 21:620-637.

1971. Euglossine bees as long distance-pollinators of tropical plants. Science 171:203-205.

Kalin A., M.T. y M. Cabrera. 1978. Preliminary self-incompatibility tests for some tropical claud forest species in Venezuela. Incompat. Newsl. 8:72-76.

LEVIN, D.A. y W.W. ANDERSON. 1970. Competition for pollinators between simultaneously flowering species. Amer. Nat. 104:455-467. 
1973. Competition for pollinator service: a stimulus for the evolution of autogamy. Evolution 26:668-669.

Mosqunn, T. 1971. Competition for pollinators as a stimulus for the evolution of flowering time. Oikos 22:398-402.

OpleR, P.A., H.G. BAKER y G.W. FrankIE. 1980. Plant reproductive characteristics during secondary succession in neotropical lowland forest ecosystems. Biotropica 12 (2, suppl.): 40-46.

POJAR, J. 1974. Reproductive dynamics of four plant communities of Southwestern British Columbia. Canad. J. Bot. 52:1819-1834.

PRIMAK, P y R.B. TOMLISON. 1980. Variation in tropical forest breeding systems. Biotropica 12:229-231.

RuIz, T. y M.T. Kalin A., 1978. Plant reproductive ecology of a secondary deciduous tropical forest in Venezuela. Biotropica 10:221-230.

Rzedowski, J. y R. Mcvaugh. 1966. La vegetación de Nueva Galicia. Contr. Univ. Mich. Herb. 9:1-123. 1970. Nota sobre el bosque mesófilo de montaña en el Valle de México. An. Esc. Nac. Cienc. Biol. (México) $18: 91-106$.

1978. La vegetación de México. Ed. Limusa, México.

THIEN, L.B. 1980. Patterns of pollination in primitive angiosperms. Biotropica 12:1-13.

WALTER, H. 1973. Vegetation of the earth. Springer-Verlag, Nueva York.

WhITEHEAD, D.R. 1969. Wind pollination in the angiosperms: evolutionary and environmental considerations. Evolution 23:28-35. 